TAMKANG JOURNAL OF MATHEMATICS

Volume 41, Number 4, 393-402, Winter 2010

\title{
A TOTAL LABELLINGS OF $m$ TRIANGLES
}

\author{
H. V. CHEN
}

\begin{abstract}
Assume that we have $m$ triangles. In this paper, we discuss certain labelling of the $m$ triangles called $c$-Erdösian for some positive integers $c$. We regard labellings of the vertices of the triangles by positive integers, which induce the edge labels for the triangles as the sum of the two incident vertex labels. They have the property that each vertex label and edge label appears only once in the set of positive integers $\{c, \ldots, c+6 m-1\}$. Here, we show how to construct certain $c$-Erdösian of $m$ triangles.
\end{abstract}

\section{Introduction}

Graph labellings are assignment of integers to the vertices or edges, or both, subject to certain conditions. In 1963, Sedláček [4] introduced magic labellings for graphs. Stewart ([7], [8]) studied various ways to label the edges of a graph. A connected graph is said to be semi-magic if there is a labelling of the edges with integers such that for each vertex $v$ the sum of the labels of all edges incident with $v$ is the same for all $v$. A semi-magic labelling where the edges are labelled with distinct positive integers is called a magic labelling. Kotzig and Rosa [1] introduced magic labellings of a graph $G(V, E)$ as a bijection $f$ from $V \cup E$ to $\{1,2, \ldots,|V \cup E|\}$ such that for all edges $x y$, $f(x)+f(y)+f(x y)$ is constant and this type of graph labelling is called edge-magic total labelling. In 1999, MacDougall, Miller, Slamin, and Wallis [2] introduced the notion of a vertex-magic total labelling. For a graph $G(V, E)$ an injective mapping $f$ from $V \cup E$ to the set $\{1,2, \ldots,|V|+|E|\}$ is a vertex-magic total labelling if there is a constant $k$, called the magic constant, such that for every vertex $v, f(v)+\sum f(v u)=k$ where the sum is over all vertices $u$ adjacent to $v$.

In this paper, we only consider a graph which consists of $m$ triangles. Let $G T_{m}=$ $\left(V_{m}, E_{m}\right)$ be the finite (disconnected) graph with vertex set $V$ of size $|V|=3 m$ and edge set $E_{m}$ of size $\left|E_{m}\right|=3 m$, consisting of $m$ disjoint triangles $K_{3}$, that is we let $G T_{m}=m \cdot K_{3}$. A total labelling of the graph $G T_{m}$ is a positive integer valued function $f: V_{m} \cup E_{m} \rightarrow N$. A labelling is said to be magic if its range consists of the integers $\{1,2, \ldots, 6 m\}$ and it is said to be $c$-magic if its range consists of the integers $\{c, c+$ $1, \ldots, c+6 m-1\}$, for any positive integer $c>0$.

Received March 1, 2010.

Key words and phrases. c-Erdösian, Skolem sequences, Langford sequences, magic labelling 
We say that $f$ is a $c$-Erdösian triangle labelling if it is $c$-magic and if it has the following property: For any edge $x y \in E_{m}$, with $x, y \in V_{m}$ we have

$$
f(x)+f(y)=f(x y) .
$$

For convenience, we say that $G T_{m}$ is $c$-Erdösian if it satisfies the conditions above.

If $x_{1}, x_{2}, \ldots, x_{3 m}$ is an enumeration of the set of vertices of $V$, then we see that the values $f\left(x_{1}\right), f\left(x_{2}\right), \ldots, f\left(x_{3 m}\right)$ are a subset of half size of the set of all the integers in $\{c, c+1, \ldots, c+6 m-1\}$. It is however necessary to point out that these values do not in general consist of the smallest integers, i.e. do not consist of the values $\{c, c+1, \ldots c+$ $3 m-1\}$. For example in the case $m=1$, the triangle $K_{3}$ with the vertices $V_{1}=\{x, y, z\}$ and the edges $E_{1}=\{x y, x z, y z\}$ is 1 -Erdösian with $f(x)=1, f(y)=2, f(z)=4$; and also 2-Erdösian with $f(x)=2, f(y)=3, f(z)=4$. Clearly in the first case we have an edge label smaller than a vertex label, namely $f(x y)=3<f(z)=4$.

Proposition 1. The cycle $K_{3}$ is 1-Erdösian and 2-Erdösian but it is not c-Erdösian for $c \geq 3$.

Proof. Let $x_{1}, x_{2}, x_{3}$ is an enumeration of the vertices of $K_{3}$. If $X$ be the sum of the vertex labels, then $X \geq c+(c+1)+(c+2)=3 c+3$. Note that the total sum of all the labels is $3 X=c+\cdots+(c+5)=3(2 c+5)$, and it follows that $X=2 c+5$. Therefore $2 c+5 \geq 3 c+3$, and hence $c \leq 2$.

Let $G T_{m}$ consists of $m$ disjoint triangles. Let $D_{i}=\left\{a_{i}, b_{i}, c_{i}, a_{i}+b_{i}, b_{i}+c_{i}, a_{i}+c_{i}\right\}$, $i=1, \ldots, m$ be their vertex and edge labels for each triangle. In other word, the system $\left\{D_{1}, \ldots, D_{m}\right\}$ is called $c$-Erdösian if its range consists of the integers $\{c, c+1, \ldots, c+6 m-$ $1\}$, for any positive integer $c>0$. For convenience, the elements of $D_{i}$ can be represented in the following form of a 2-by-3 array, where the top row indicates the vertex labels and the bottom row shows the edge labels:

$$
\begin{array}{ccc}
a_{i} & b_{i} & c_{i} \\
a_{i}+b_{i} & a_{i}+c_{i} & b_{i}+c_{i}
\end{array}
$$

We first look at the following necessary condition:

Proposition 2. If $G T_{m}$ is c-Erdösian, then $c \leq \frac{3 m+1}{2}$.

Proof. Let $D_{i}$ be the set of vertex and edge labels for $i$-th triangle where $D_{i}=$ $\left\{a_{i}, b_{i}, c_{i}, a_{i}+b_{i}, b_{i}+c_{i}, a_{i}+c_{i}\right\}, i=1,2, \ldots, m$. Note that $D_{1} \cup \cdots \cup D_{m}=\{c, c+$ $1, \ldots, c+6 m-1\}$ and $c+\cdots+(c+6 m-1)=6 m c+3 m(6 m-1)$. Let $T S$ be the sum of the vertices and $B S$ be the sum of edges. Then, $T S=a_{i}+b_{i}+c_{i}$ and $B S=\left(a_{i}+b_{i}\right)+\left(a_{i}+c_{i}\right)+\left(b_{i}+c_{i}\right)=2 T S$ for all $i=1, \ldots, m$. Note that

$$
T S \geq c+(c+1)+\cdots+[c+(3 m-1)]=3 m c+\frac{3 m}{2}(3 m-1)
$$


and

$$
2 T S=B S \leq(c+3 m)+\cdots+[c+(6 m-1)]=3 m c+\frac{3 m}{2}(9 m-1) .
$$

Then $T S \leq \frac{3 m c}{2}+\frac{3 m}{4}(9 m-1)$ and it follows that

$$
3 m c+\frac{3 m}{2}(3 m-1) \leq \frac{3 m c}{2}+\frac{3 m}{4}(9 m-1) .
$$

Therefore $c \leq \frac{3 m+1}{2}$.

Proposition 3. For all integers $h \geq 0$ and $c=3 h+2$, there exists a $G T_{2 h+1}$ which is c-Erdösian.

Proof. Let $m=2 h+1$. Since $h \geq 0$ and $c=3 h+2$, we have

$$
D_{1} \cup \cdots \cup D_{m}=\{c, \ldots, c+6 m-1\}=\{3 h+2, \ldots, 15 h+7\} .
$$

All the integers in the set $\{3 h+2, \ldots, 15 h+7\}$ can be partitioned into six intervals of length $m$ each. Note that there exist three permutation vectors of length $m, \pi_{1}=$ $(-c+1,-c+2, \ldots,-1,0,1, \ldots, c-2, c-1), \pi_{2}=(c-1, c-3, \ldots,-c+1, c-2, c-$ $4, \ldots,-c+4,-c+2)$ and $\pi_{3}=(0,1, \ldots, c-1,-c+1,-c+2, \ldots,-2,-1)$ which satisfy the equation $\pi_{1}+\pi_{2}+\pi_{3}=\tilde{0}$. Furthermore $\pi_{1}+\pi_{2}, \pi_{1}+\pi_{3}$ and $\pi_{2}+\pi_{3}$ are the permutation vectors which consist of integers from the set $\{-c+1,-c+2, \ldots,-1,0,1,2, \ldots, c-$ $2, c-1\}$. By using the permutation vectors, we are able to construct a $G T_{2 h+1}$ which is $c$-Erdösian. Note that in this case, we have $\cup_{i=1}^{m}\left\{a_{i}, b_{i}, c_{i}\right\}=\{c, \ldots, c+3 m-1\}$ and $\cup_{i=1}^{m}\left\{a_{i}+b_{i}, a_{i}+c_{i}, b_{i}+c_{i}\right\}=\{c+3 m, \ldots, c+6 m-1\}$.

The following example shows the construction of $G T_{2 h+1}$ which is $c$-Erdösian.

Example 1. If $h=2$, then $m=5$ and $c=8$. Let $\pi_{1}=(-2,-1,0,1,2), \pi_{2}=$ $(1,2,-2,-1,0)$ and $\pi_{3}=(1,-1,2,0,-2)$. Then we obtain that $\pi_{1}+\pi_{2}=(-1,1,-2,0,2)$, $\pi_{1}+\pi_{3}=(-1,-2,2,1,0)$ and $\pi_{2}+\pi_{3}=(2,1,0,-1,-2)$ which are the permutation vectors with the integers $\{-2,-1,0,1,2\}$. It is clear that $\pi_{1}+\pi_{2}+\pi_{3}=\tilde{0}$. All the vertex labels in a triangle will be assigned according to the three permutation vectors above. Let $\pi_{i}=\left(p_{1}, p_{2}, p_{3}, p_{4}, p_{5}\right)$ be the permutation vectors as mentioned above for $i=1,2,3$. One of the vertex labels in the $j$-th triangle is indicated by the value of $p_{j}$ for $j=1,2, \ldots, 5$. We shall assign the integers $\{8,9,10,11,12\}$ as the first vertex labels in the five triangles according to the entries $p_{j}$ of the permutation vector $\pi_{1}$. Since $\pi_{1}=(-2,-1,0,1,2)$ and the smallest integer in $\{8,9,10,11,12\}$ is 8,8 is assigned as one of the vertex labels in the first triangle. It follows that 12 is assigned as one of the vertex labels in the fifth triangle since the entries in $\pi_{1}$ is increasing from -2 to 2 . The next five integers for the second vertex labels include $\{13,14,15,16,17\}$ and we have $\pi_{2}=(1,2,-2,-1,0)$. Since $p_{3}=-2$ in $\pi_{2}$, we note that the second vertex labels for the third triangle is 13 . Hence, the second vertex labels in the forth triangle is 14 , fifth triangle is 15 , first triangle is 16 and second triangle is 17, respectively. By using the similar argument, we can assign 
the third vertex labels for the five triangles from the set of integers $\{18,19,20,21,22\}$, according to the entries of $\pi_{3}$. After we have all the vertex labels, the edge labels can be obtained by summing up the values of the vertex labels. Hence, we have the following 8-Erdösian.

$\begin{array}{cccc}81621 & 91719 & 101322 \\ 242937, & 262836, & 233235, \\ 11 & 1420 & 121518 \\ 253134, & 273033 .\end{array}$

We end the first section by the following straightforward result.

Proposition 4. If $G T_{m}$ is 4-Erdösian, then there exists a $G T_{m+1}$ which is 1-Erdösian.

Proof. Let $D_{0}=\{1,2,3,6 m+4,6 m+5,6 m+6\}$. Since $D=\{4,5, \ldots, 6 m+3\}$, we obtain a $G T_{m+1}$ which is 1-Erdösian by considering $D_{0} \cup D$.

Example 2. Given a $G T_{7}$ which is 4-Erdösian as follows:

\begin{tabular}{|c|c|c|}
\hline 41728 & $\begin{array}{lll}5 & 1529\end{array}$ & 61330 \\
\hline 213245 , & 203444 , & 193643 , \\
\hline 81625 & 91426 & 101227 \\
\hline 243341 , & 233540 , & 223739 . \\
\hline
\end{tabular}

We can always construct a 2-by-3 array

1462

47348

and hence there exists a $G T_{8}$ which is 1-Erdösian.

\section{Some Special Classes of Triangle Labellings}

We now specify various classes of special $c$-Erdösian of $G T_{m}$. As a standard notation we let the vertex labels be

$$
a_{i}<b_{i}<c_{i} \text { for } i=1,2, \ldots, m
$$

Note that the three edge labels then become

$$
a_{i}+b_{i}<a_{i}+c_{i}<b_{i}+c_{i} \text { for } i=1,2, \ldots, m
$$

With this notation we now give a list of several conditions on the various vertex and edge labels. 
(i) This condition says that the vertex labels in position $a_{i}$ form the first run of integers:

$$
\left\{a_{1}, a_{2}, \ldots, a_{m}\right\}=\{c, c+1, \ldots, c+m-1\} .
$$

(ii) This condition says that the vertex labels in position $a_{i}, b_{i}$ together form the first two runs of integers:

$$
\left\{a_{1}, a_{2}, \ldots, a_{m}\right\} \cup\left\{b_{1}, b_{2}, \ldots, b_{m}\right\}=\{c, c+1, \ldots, c+2 m-1\} .
$$

(iii) This condition says that the vertex labels in position $a_{i}, b_{i}$ and the edge label in position $a_{i}+b_{i}$ form the first three runs of integers:

$$
\left\{a_{i}, b_{i}, a_{i}+b_{i}: i=1,2, \ldots, m\right\}=\{c, c+1, \ldots, c+3 m-1\} .
$$

Proposition 5. For all $c \geq 1$, there exists a $G T_{2 c-1}$ which is $c$-Erdösian and satisfies the condition (iii).

Proof. Let $\pi_{1}$ be the identity permutation of integers from $\{-c+1, \ldots, c-1\}$, i.e., $\pi_{1}=(-c+1,-c+2, \ldots,-1,0,1, \ldots, c-2, c-1)$. Then there exist permutations $\pi_{2}=$ $(c-1, c-3, \ldots,-c+1, c-2, c-4, \ldots,-c+4,-c+2)$ and $\pi_{3}=(0,1, \ldots, c-1,-c+$ $1,-c+2, \ldots,-2,-1)$ such that $\pi_{1}+\pi_{2}, \pi_{1}+\pi_{3}$ and $\pi_{2}+\pi_{3}$ are also permutations of integers from $\{-c+1, \ldots, c-1\}$. Note that

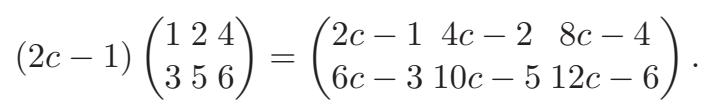

Then we obtain the following 2-by-3 array which $e_{(i) ; j}$ is the element of $\pi_{i}$ and $e_{[(i)+(k)] ; j}$ is the element of $\pi_{i}+\pi_{k}$ at the $j$-th position of the permutation vector, respectively.

$$
\begin{array}{ccc}
e_{(1) ; j} & e_{(2) ; j} & e_{(3) ; j} \\
e_{[(1)+(2)] ; j} & e_{[(1)+(3)] ; j} & e_{[(2)+(3)] ; j},
\end{array}
$$

$j=1, \ldots, 2 c-1$. By adding each element in respective position in (1.1) and (1.2), we have $D_{j}=\left\{e_{(1) ; j}+(2 c-1), e_{(2) ; j}+(4 c-2), e_{(3) ; j}+(8 c-4), e_{[(1)+(2)] ; j}+(6 c-3), e_{[(1)+(3)] ; j}+(10 c-\right.$ $\left.5), e_{[(2)+(3)] ; j}+(12 c-6)\right\}, j=1,2, \ldots, 2 c-1$. Hence, $\cup_{j=1}^{2 c-1} D_{j}=\{c, c+1, \ldots, 13 c-7\}$.

The following example presents the construction of $c$-Erdösian $m$ disjoint triangles described in the Proposition 5.

Example 3. If $c=2$, we have $\pi_{1}=(-1,0,1), \pi_{2}=(1,-1,0)$ and $\pi_{3}=(0,1,-1)$. Then

$$
\begin{array}{ccccccccccc}
-1 & 1 & 0 & & 0 & -1 & 1 & & 1 & 0 & -1 \\
0 & -1 & 1, & & -1 & 1 & 0, & & 1 & 0 & -1 .
\end{array}
$$

Since

$$
(2 c-1)\left(\begin{array}{lll}
1 & 2 & 4 \\
3 & 5 & 6
\end{array}\right)=\left(\begin{array}{lll}
3 & 6 & 12 \\
9 & 15 & 18
\end{array}\right)
$$

then the 2-Erdösian of $G T_{3}$ is as follows:

$$
\begin{aligned}
& \begin{array}{lllllllll}
2 & 7 & 12 & 3 & 5 & 13 & 4 & 6 & 11
\end{array} \\
& 91419, \quad 81618, \quad 101517 .
\end{aligned}
$$


We can also formulate the dual conditions to (i) and to (ii), and some other conditions like (v).

(iv) This condition says that the edge labels in position $b_{i}+c_{i}$ form the last run of integers:

$$
\left\{b_{1}+c_{1}, b_{2}+c_{2}, \ldots, b_{m}+c_{m}\right\}=\{c+5 m, c+5 m+1, \ldots, c+6 m-1\} .
$$

(v) This condition says that the edge labels in position $b_{i}$ form precisely the second run of integers:

$$
\left\{b_{1}, b_{2}, \ldots, b_{m}\right\}=\{c+m, c+m+1, \ldots, c+2 m-1\} .
$$

Proposition 6. Assume that $G T_{m}$ is c-Erdösian that satisfies the condition (ii). Then it also has the properties

$$
\begin{aligned}
\left\{a_{i}+b_{i}, c_{i}: i\right. & =1,2, \ldots, m\}=\{c+2 m, c+2 m+1, \ldots, c+4 m-1\} \\
\left\{a_{i}+c_{i}, b_{i}+c_{i}: i\right. & =1,2, \ldots, m\}=\{c+4 m, c+4 m+1, \ldots, c+6 m-1\} .
\end{aligned}
$$

In particular it satisfies property $(v)$.

Proof. Note that $(c+2 m)+\cdots+(c+4 m-1)=m(2 c+6 m-1)$. Hence $\sum_{i=1}^{m}\left(a_{i}+b_{i}+c_{i}\right)=$ $m(2 c+6 m-1)$, it follows that $\left\{a_{i}+b_{i}, c_{i}: i=1,2, \ldots, m\right\}=\{c+2 m, c+2 m+1, \ldots, c+$ $4 m-1\}$. Since $(c+4 m)+\cdots+(c+6 m-1)=m(2 c+10 m-1)$ and $2 \sum_{i=1}^{m}\left[\left(a_{i}+b_{i}\right)+c_{i}\right]=$ $2 m(2 c+6 m-1)$, we have $\sum_{i=1}^{m}\left(a_{i}+b_{i}\right)+2 \sum_{i=1}^{m} c_{i}=m(2 c+10 m-1)$.

\section{Constant sum of the vertex labels}

Proposition 7. If the sum of the vertex labels over any one triangle is a constant, then

$$
a_{i}+b_{i}+c_{i}=2 c+6 m-1 \text { for all } i=1,2, \ldots, m .
$$

Proof. The constant in (1.3) is obtained by summing over all labels,

$$
c+(c+1)+\cdots+(c+6 m-1)=6 m c+3 m(6 m-1)=3 m(2 c+6 m-1)
$$

and then dividing this expression by $3 m$, since there are $m$ triangles and each triangle has total sum of labels $3\left(a_{i}+b_{i}+c_{i}\right)$.

The following is an example which the sum of the vertex labels over each triangle which is all distinct.

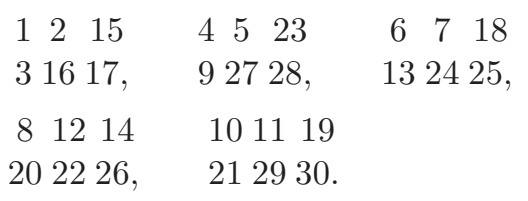

To construct our $c$-Erdösian of $m$ triangles, we will use Skolem and Langford sequences. A Skolem sequence of order $n$ is a sequence $S=\left(s_{1}, s_{2}, \ldots, s_{2 n}\right)$ of $2 n$ integers satisfying the conditions 
(S1) for every $k \in\{1,2, \ldots, n\}$ there exist exactly two elements $s_{i}, s_{j} \in S$ such that $s_{i}=s_{j}=k$;

(S2) if $s_{i}=s_{j}=k$ with $i<j$, then $j-i=k$.

Skolem sequences can also be written as collections of ordered pairs $\left\{\left(a_{i}, b_{i}\right): 1 \leq i \leq\right.$ $\left.n, b_{i}-a_{i}=i\right\}$ with $\sum_{i=1}^{n}\left\{a_{i}, b_{i}\right\}=\{1,2, \ldots, 2 n\}$. A hooked Skolem sequence of order $n$ is a sequence $S=\left(s_{1}, s_{2}, \ldots, s_{2 n+1}\right)$ of $2 n+1$ integers satisfying the conditions (S1) and (S2) above and

(S3) $s_{2 n}=0$.

Skolem sequences can also be written as collections of ordered pairs $\left\{\left(p_{i}, q_{i}\right): 1 \leq i \leq\right.$ $\left.n, q_{i}-p_{i}=i\right\}$ with $\sum_{i=1}^{n}\left\{p_{i}, q_{i}\right\}=\{1,2, \ldots, 2 n\}$.

A Langford sequence of order $n$ and defect $d, n>d$, is a sequence $L=\left(l_{1}, l_{2}, \ldots, l_{2 n}\right)$ of $2 n$ integers satisfying the conditions

(L1) for every $k \in\{d, d+1, \ldots, d+n-1\}$ there exist exactly two elements $l_{i}, l_{j} \in L$ such that $l_{i}=l_{j}=k$, and

(L2) if $l_{i}=l_{j}=k$ with $i<j$, then $j-i=k$.

The hooked Langford sequences of order $n$ and defect $d$ is a sequence $L=\left(l_{1}, l_{2}, \ldots, l_{2 n+1}\right)$ of $2 n+1$ integers satisfying conditions (L1) and (L2) above and

(L3) $l_{2 n}=0$.

Langford sequences are also written as collections of ordered pairs $\left\{\left(p_{i}, q_{i}\right): d \leq i \leq\right.$ $\left.d+n-1, q_{i}-p_{i}=i\right\}$ with $\sum_{i=1}^{n}\left\{p_{i}, q_{i}\right\}=\{d, d+1, \ldots, d+2 n-1\}$.

Clearly, a (hooked) Langford sequence with defect 1 is a (hooked) Skolem sequence. It is well-known that a Skolem sequence of order $n$ exists if and only if $n \equiv 0,1(\bmod$ 4) [6] and a hooked Skolem sequence of order $n$ exists if and only if $n \equiv 2,3(\bmod 4)$ [3]. The following theorem gives necessary and sufficient conditions for the existence of Langford sequences.

Theorem 1. ([5]) A Langford sequence of order $n$ and defect $d$ exists if and only if

(i) $n \geq 2 d-1$, and

(ii) $n \equiv 0,1(\bmod 4)$ and $d$ is odd, or $n \equiv 2,3(\bmod 4)$ and $d$ is even.

A hooked Langford sequence of order $n$ and defect $d$ exists if and only if

(i) $n(n-2 d+1)+2 \geq 0$, and

(ii) $n \equiv 2,3(\bmod 4)$ and $d$ is odd, or $n \equiv 1,2(\bmod 4)$ and $d$ is even. 
The set of positive integers $\{c, c+1, \ldots, c+3 m-1\}$ can be decomposed into $m$ disjoint triples of the form $\left\{a_{i}, b_{i}, a_{i}+b_{i}\right\}$ with $i=1,2, \ldots, m$. Given a collection of ordered pairs of Langford Sequence of order $m,\left\{\left(p_{i}, q_{i}\right): c \leq i \leq c+m-1, q_{i}-p_{i}=i\right\}$ with $\sum_{i=1}^{m}\left\{p_{i}, q_{i}\right\}=\{c, c+1, \ldots, c+2 m-1\}$. We obtain the following triples $\left\{\left(i, p_{i}+\right.\right.$ $\left.\left.m+1, q_{i}+m+1\right): c \leq i \leq c+m-1, q_{i}-p_{i}=i\right\}$. For example, given a Langford sequence of order 5 and defect $3, L=(7,5,3,6,4,3,5,7,4,6)$, we obtain the collection of ordered pairs and triples respectively, as follows:

$$
\begin{gathered}
\{(3,6),(5,9),(2,7),(4,10),(1,8)\} ; \\
\{(3,10,13),(4,12,16),(5,9,14),(6,11,17),(7,8,15)\} .
\end{gathered}
$$

The hooked Langford sequences is defined similarly.

In the next example, we show how to use the triples that we obtain from Langford sequences to construct $m$ disjoint triangles which is $c$-Erdösian.

Example 4. Given a Langford sequence of order 3 and defect $c=2, L=(3,4,2,3,2,4)$, we rewrite in triples as $\{(2,7,9),(3,5,8),(4,6,10)\}$. The first two integers in each triple are the vertex labels and the third vertex label can be obtained by using Proposition 7 . So the 2-Erdösian of $G T_{3}$ is clear from the following 2-by-3 arrays:

$$
\begin{aligned}
& \begin{array}{lllllllll}
2 & 7 & 12 & 3 & 5 & 13 & 4 & 6 & 11
\end{array} \\
& 91419, \quad 81618, \quad 101517 .
\end{aligned}
$$

We next present a theorem involving the construction of $c$-Erdösian $m$ disjoint triangles by using the Langford sequences or hooked Langford sequences.

Theorem 2. For $m \equiv 0,1(4)$ and for any $1 \leq c \leq \frac{m+1}{2}$ the graph $G T_{m}$ consisting of $m$ disjoint copies of the triangle $K_{3}$ is c-Erdösian.

Proof. Let $\left(x_{i}, y_{i}\right), i=1, \ldots, m$ be the collection of ordered pairs of Langford sequences (or hooked Langford sequences) where $m \equiv 0,1(4)$ and it is clear that $\left\{y_{i}-x_{i}: i=\right.$ $1, \ldots, m\}=\{c, \ldots, c+m-1\}$. Let $a_{i}=y_{i}-x_{i}, b_{i}=x_{i}+m$ and $c_{i}=2 c+6 m-1-\left(a_{i}+b_{i}\right)$ then the result follows from Theorem 1.

Example 5. From the collection of ordered pairs of hooked Langford sequence $m=5$ and $c=2$, i.e.,

$$
\{(2,4),(7,10),(5,9),(3,8),(6,12)\},
$$

\begin{tabular}{|c|c|}
\hline $\begin{array}{lll}2 & 7 & 24\end{array}$ & $\begin{array}{lll}3 & 12 & 18\end{array}$ \\
\hline 92631 , & 152130 \\
\hline $\begin{array}{ccc}5 & 8 & 20 \\
1 & 2 & 5\end{array}$ & $\begin{array}{llll}6 & 11 & 16\end{array}$ \\
\hline 32528 & 172227 . \\
\hline
\end{tabular}

we obtain the triples as follows:

$$
\{(2,7,9),(3,12,15),(4,10,14),(5,8,13),(6,11,17)\} .
$$

Since $c_{i}=2(2)+6(5)-1-a_{i}-b_{i}=33-a_{i}-b_{i}$, then we have the following 2-Erdösian arrays of $G T_{5}$ : 
Proposition 8. Assume that $G T_{m}$ is c-Erdösian that satisfies the condition (iii). The set of positive integers $\{c, c+1, \ldots, c+3 m-1\}$ can be decomposed into $m$ disjoint triples of the form $\left\{a_{i}, b_{i}, a_{i}+b_{i}\right\}$ with $i=1,2, \ldots, m$. In addition, the sum of the third vertex labels $c_{1}+c_{2}+\cdots+c_{m}$ takes the value

$$
\sum_{i=1}^{m} c_{i}=\frac{m}{4}(2 c+15 m-1) .
$$

The set of values $\left\{c_{i}, a_{i}+c_{i}: i=1,2, \ldots, m\right\}$ constitutes the fourth and fifth run of the integers in $\{c, \ldots, c+6 m-1\}$, that is

$$
\left\{c_{i}, a_{i}+c_{i}: i=1,2, \ldots, m\right\}=\{c+3 m, c+3 m+1, \ldots, c+5 m-1\},
$$

and hence it satisfies (iv), that is the integers $\left\{b_{i}+c_{i}: i=1,2, \ldots, m\right\}$ constitute the top run of integers

$$
\left\{b_{i}+c_{i}: i=1,2, \ldots, m\right\}=\{c+5 m, c+5 m+1, \ldots, c+6 m-1\} .
$$

Proof. Since $c_{i}=2 c+6 m-1-\left(a_{i}+b_{i}\right)$ and $\sum_{i=1}^{m}\left(a_{i}+b_{i}\right)=\frac{3 m}{4}(2 c+3 m-1)$, it is clear that $\sum_{i=1}^{m} c_{i}=\frac{m}{4}(2 c+15 m-1)$.

Proposition 9. For all $m \geq 1$, there exists a 1-Erdösian $G T_{m}$ that satisfies the conditions (i) and (iii).

Proof. Let $g_{i}, h_{i}$ be distinct integers for all $i=1, \ldots, m$. By using the Skolem sequences or hooked Skolem sequences, we are able to get $h_{i}=g_{i}+i$ for all $i=1, \ldots, m$. Note that we obtain a 1-Erdösian $G T_{m}$ as follows:

$$
\begin{array}{ccc}
i & g_{i} & k_{i} \\
h_{i} i+k_{i} & g_{i}+k_{i}
\end{array}
$$

\begin{tabular}{|c|c|}
\hline & $\begin{array}{lll}2 & 6 & 17 \\
\end{array}$ \\
\hline 142 & 81923 \\
\hline
\end{tabular}

for all $i=1, \ldots, m$, and

$$
i+g_{i}+k_{i}=\frac{1+\cdots+6 m}{3 m}=\frac{6 m}{2}(1+6 m)\left(\frac{1}{3 m}\right)=1+6 m .
$$

Example 6 (i) From the Skolem sequence of order 4 in triples $\{(1,11,12),(2,6,8),(3,7,10),(4,5,9)\}$, we obtain the following 1-Erdösian of $G T_{4}$ :

(ii) Given a hooked Skolem sequence of order 6 in triples $\{(3,16,19),(6,11,17),(5,10,15),(2,12,14),(4,9,13),(1,7,8)\}$, we obtain a 1-Erdösian of $G T_{6}$ as follows:

$\begin{array}{cccccccc}1729 & 212 & 23 & 31618 & 4 & 9 & 24 \\ 83036, & 142535, & 192134, & 1328 & 33, \\ 5 & 1022 & 6 & 1120\end{array}$


Proposition 10. Assume that $G T_{m}$ is c-Erdösian that satisfies the conditions (i) and (iii). The set of positive integers $\{c, c+1, \ldots, c+3 m-1\}$ can be decomposed into $m$ disjoint triples of the form $\left\{a_{i}, b_{i}, a_{i}+b_{i}\right\}$ and also the set of $m$ triples $\left\{a_{i}, c_{i}-\right.$ $\left.2 m, a_{i}+c_{i}-2 m\right\}$, respectively, for $i=1,2, \ldots, m$. The two triples $\left\{a_{i}, b_{i}, a_{i}+b_{i}\right\}$ and $\left\{a_{i}, c_{i}-2 m, a_{i}+c_{i}-2 m\right\}$ satisfy the following comparability property:

The sequence of sums of the middle values corresponding to the same difference $a_{i}$ forms a run of integers:

$$
\left\{b_{i}+c_{i}-2 m: i=1,2, \ldots, m\right\}=\{c+3 m, c+3 m+1, \ldots, c+4 m-1\} .
$$

Proof. Note that $\sum_{i=1}^{m} a_{i}=\frac{m}{2}(2 c+m-1), \sum_{i=1}^{m}\left(a_{i}+b_{i}+c_{i}\right)=m(2 c+6 m-1)$ and $\sum_{i=1}^{m} a_{i}+b_{i}=\frac{3 m}{4}(2 c+3 m-1)$. Since $\sum_{i=1}^{m} c_{i}=\frac{m}{4}(2 c+15 m-1)$, we have $\sum_{i=1}^{m}\left[2\left(a_{i}+c_{i}\right)-4 m\right]=\frac{3 m}{2}(2 c+3 m-1)=c+\cdots+(c+3 m-1)$. Hence, The set of positive integers $\{c, c+1, \ldots, c+3 m-1\}$ can be decomposed into $m$ disjoint triples of the form $\left\{a_{i}, c_{i}-2 m, a_{i}+c_{i}-2 m\right\}$.

Proposition 11. Assume that $G T_{m}$ is c-Erdösian that satisfies the conditions (i) and (ii). It trivially also satisfies condition (v). Then the function $\pi\left(a_{i}\right)=b_{i}-m$ is a permutation of the set $\{c, c+1, \ldots, c+m-1\}$.

Proof. Since $c+m \leq b_{i} \leq c+2 m-1, i=1, \ldots, m$ and $b_{i} \neq b_{j}$ for all $i \neq j$, we have $c \leq b_{i}-m \leq c+m-1$. Then $\pi\left(a_{i}\right)=b_{i}-m$ is a permutation of the set $\{c, c+1, \ldots, c+m-1\}$.

\section{Acknowledgement}

The author wishes to thank Dr Thomas Bier for his assistance in this research.

\section{References}

[1] A. Kotzig and A. Rosa, Magic valuations of finite graphs, Canad. Math. Bull., 13 (1970), 451-461.

[2] J. A. MacDougall, M. Miller, Slamin, and W. D. Wallis, Vertex-magic total labellings of graphs, Util. Math., 61 (2002), 3-21.

[3] E. S. O'Keefe, Verification of a conjecture of Th Skolem, Math. Scand., 9(1961), 80-82.

[4] J. Sedláček, Problem 27, in Theory of Graphs and its Applications, Proc. Symposium Smolenice, June, (1963), 163-167.

[5] J. E. Simpson, Langford sequences: perfect and hooked, Discrete Math., 44(1983), 97-104.

[6] Th. Skolem, On certain distributions of integers in pairs with given difference, Math. Scand., 5(1957), 57-68.

[7] B. M. Stewart, Magixc graphs, Canadian J. Math., 18(1966), 1031-1059.

[8] B. M. Stewart, Supermagic complete graphs, Canadian J. Math., 19(1967), 427-438.

Department of Mathematical Actuarial Sciences, Faculty of Engineering and Science, Universiti Tunku Abdul Rahman, Jalan Genting Kelang, Setapak 53300, Kuala Lumpur, Malaysia.

E-mail: chenhv@utar.edu.my 